\title{
Android Based Area Web Monitoring
}

\author{
Bayu Kanigoro, Afan Galih Salman, Jurike V Moniaga, Eric Chandra, and Zein Rezky Chandra \\ Computer Science Program, School of Computer Science, Bina Nusantara University, Indonesia
}

\begin{abstract}
The research objective is to develop an application that can be used in the monitoring of an area by using a webcam. It aims to create a sense of security on the user's application because it can monitor an area using mobile phone anywhere. The results obtained in this study is to create an area with a webcam monitoring application that can be accessed anywhere as long as the monitoring results have internet access and can also be accessed through Android Based Mobile Phone.
\end{abstract}

\section{Introduction}

In the era of globalization, developments in information technology plays an important role in various sectors of human life and mobile phone is a main actor in this era. When it was becomes popular around 1990, it was only be used for voice communication between persons but now it has a very sophisticated ability which formerly could only be done by computers which can be done by mobile phone today which is known by the wider community as a Smartphone.

One of the mobile operating system mostly used by Smartphone is Android. It is an operating system for Linux-based Smartphone. One of the advantages of Android operating system than others is open source nature so people can customize the features, which did not exist before. Virtually, Android has unlimited capabilities due to a lot people develop Android source code.

The rapid development of the computer world makes a variety of technologies accessible to the public, such as the example of security camera. Security cameras provide safety in humans due to monitor and keep an eye on things which user do not need to have in place but just by looking at a computer connected to the security camera.

The problems re-emerged as a personal computer or laptop is considered too large so that the use of security camera becomes less practical. These problems can be overcome by using the Android Smartphone that serves as a controller of a webcam so people can conduct mobile monitoring.

This paper discuss on developing Android application on mobile device, which can communicate and control some computer feature including webcam monitoring and motion detection. The prototype has limitation, which uses Android simulator to run the application and using localhost as communication network

\section{Recent Works}

There are some discussions about web monitoring. Details of the development of a simple webcam joystick, a wireless, or rather cable less, and contactless pointing device by using a webcam and a simple flexible nonelectronic joystick for recording patient movement has been described in [1].

An efficient Omni directional surveillance system for digital home security was proposed in [5]. In this surveillance system, the Omni directional scenes in a room, kitchen, car porch, dining hall, corridor, garage or backyard within a digital home are first captured using a web camera attached to a hyperbolic optical mirror. The captured scenes are then fed into a laptop computer for image processing and alarm purposes.

Another paper describes a software and hardware design solution of an embedded web-based remote monitoring system for the environment in the laboratories. Build an embedded web server to publish the data of sensor networks and video images to achieve remote monitoring, which is based on $\mathrm{B} / \mathrm{S}$ architecture. Managers can control the equipments in the lab through a web browser, which is cross-platform. The embedded database manages the data collected by sensor networks, realizing the local management of environmental data [4].

Network Video Monitoring described in [3] has been developed in the embedded field; however, current video surveillance is too expensive to limit use widely. A new method proposed in [3] uses ARM9 processor as hardware platform and embedded Linux system as software development, with USB camera as video gather. Using TCP/IP agreement realize network communication. The system inside embeds web server so users can visit resources by browser. The whole system realizes environmental monitoring and has a high performance and low cost. 
WebNet System developing by a few WebCam and wireless sensors, applying family safety and staff tracking through computers or Mobil phones has been proposed in [2]. WebNet System uses mainly ZigBee Sensor and ZigBee Location Node to build indoor tracking location system, sending ZigBee information to WebNet through ZigBee gateway Interface. WebNet controls the current status and analyze the relative positions and start WebCam to track in the mean time. WebNet sends out SMS or emails to the house owner who can remote control through computers or Mobil phones if emergency. WebNet and traditional surveillance system and the result shows that this research has an outstanding out coming were compared in [2].

\section{Design}

The design of a webcam monitoring using client/server based on 3-tier architecture is presented in figure 1. It has three important processes that a client computer runs Graphical User Interface logic, server runs business logic and it has database system as depicted in figure 1 .

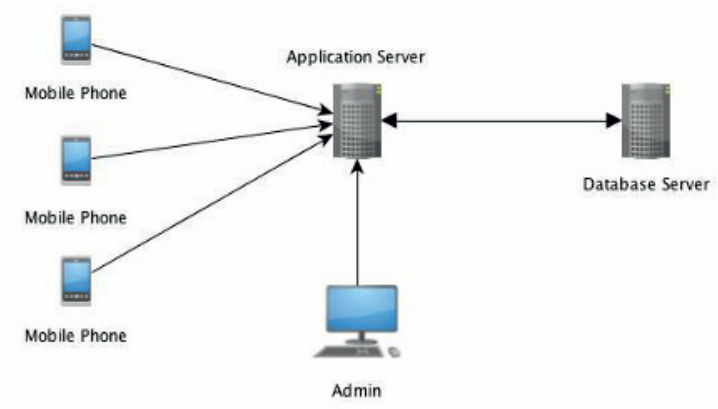

Fig. 1. Three-Tier Web Monitoring Application

The benefit of 3-tier architecture is moving the load to application server then it does not have a problem when a lot of client connected to it because in database side only one connection connected to it. The architecture can be called as fat server architecture.

The webcam monitoring application consists of client side and server side. The client is Android Smartphone, which only receive streaming data from server side. The server is webcam connected to the server and streams its data to the network. Class diagram of the design is shown in figure 2 which depict eleven classes. Most classes such as LoginActivity, Admin, Configuration, Record, Schedule, VideoLibrary, ImageCapture, StreamingActivity, Motion, and ConnectCamera are designed to be implemented as server side. Mobile_user class is implemented as client side.

\section{Implementation}

The requirement of the system uses a computer that has specification mentioned in table 1. The computer mentioned in table 1 was used as server server side including a Web Camera that was connected to USB port of the computer. Then, video streaming from Web Camera is streamed through Internet using Ethernet LAN card connected to broadband router.
Table 1. Minimum System Requirement

\begin{tabular}{|c|c|}
\hline Kind & Type \\
\hline Processor & $\begin{array}{c}\text { Intel Core i3-2310M } \\
2,10 \mathrm{GHz}\end{array}$ \\
\hline Memory & $4 \mathrm{~GB}$ \\
\hline Hard disk & $120 \mathrm{~GB}$ \\
\hline
\end{tabular}

Table 2. Minimum Mobile Device Requirement

\begin{tabular}{|c|c|}
\hline Kind & Type \\
\hline Processor & $\begin{array}{c}\text { Dual or Quad Core } \\
\text { Mobile Processor }\end{array}$ \\
\hline Memory & $\begin{array}{c}\text { 1 GB RAM with } \\
\text { 16 GB Internal } \\
\text { Storage }\end{array}$ \\
\hline Network & $\begin{array}{c}\text { 3G Network with } \\
\text { HSDPA supported }\end{array}$ \\
\hline Display & $720 \times 1080$ \\
\hline Mobile OS & Android 4.0 \\
\hline
\end{tabular}

\subsection{Server Side}

There are some functions included in server side application. The functions are Administrator login, Sequence Streaming, Record Admin, Active Camera, Motion Detection, Take Picture and Configuration.

Administrator login, Administrator runs the program in server then enter username and password for the user that be approved by administrator. After administrator has clicked login button then user data are stored and verified by administrator.

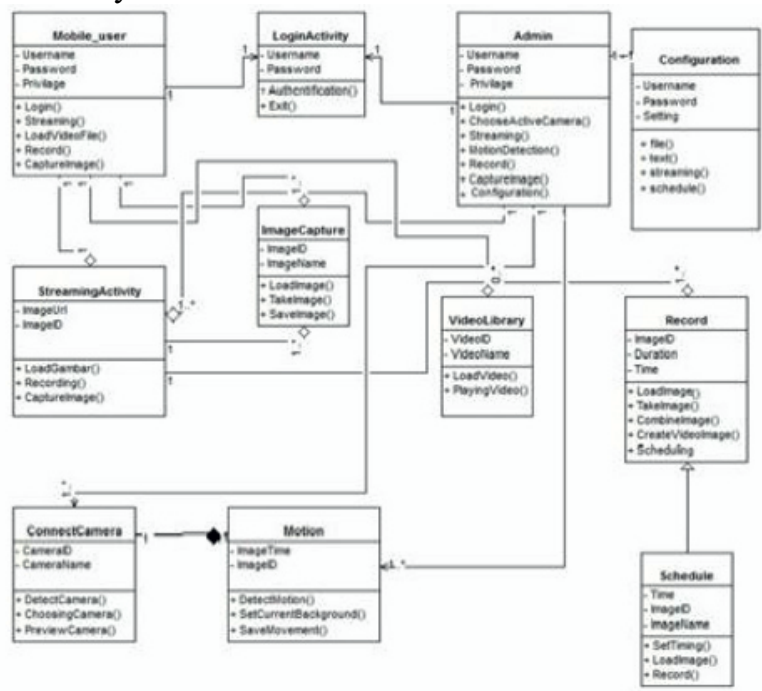

Fig. 2. Class Diagram of Webcam Monitoring System

For Sequence Streaming, Administrator runs streaming function from server by clicking Streaming Live Camera. After clicking the button, it can streams from computer or laptop camera. Administrator can record the stream when streaming by clicking take picture button. To exit from the admin application, administrator just click exit button. 
Record admin function can do record command when streaming. Administrator can command record live streaming then the result from streaming is stored based on record ID to be given and confirmed by administrator.

Administrator can choose Active Camera and give privileges to which camera is active. Administrator can choose Active Camera and give privileges to which camera is active. Administrator runs active camera list command and choose which camera to be running. Administrator runs active camera list command and choose which camera to be running.

Motion detection depicted in figure 4, administrator connect to client and give open connection command then start connection. When connected to the client, admin can close the connection by giving end connection command.

Take picture, administrator can take a picture when streaming by giving picture command and stored based on image ID. Administrator can delete the picture by giving delete image command.

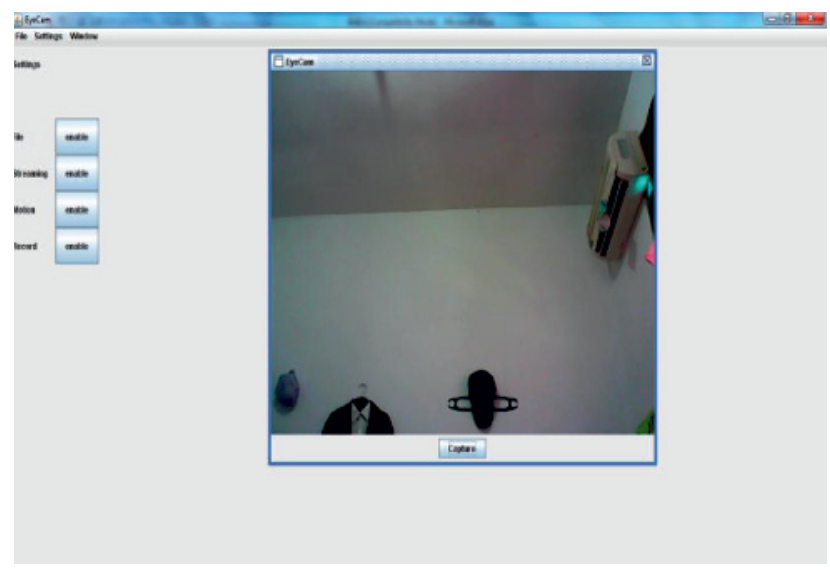

Fig. 3. Motion Detection and Scheduling

Configuration, administrator can control preference of the application. The configuration can control file, text, streaming password, scheduling and startup.

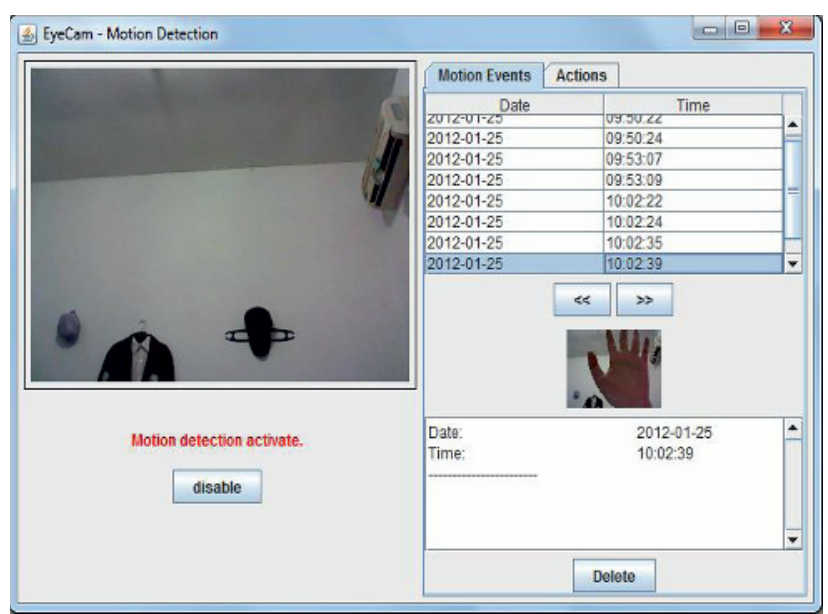

Fig. 6. Windows Capture
Login Mobile, user runs the application on Android by entering username and password based on user profile and clicked login button. After clicked the button, user data profile is stored, authenticated and verified.

Streaming Mobile, user receives streaming video from server by clicking Streaming Live Camera and watches the video from Android Smartphone. User can record the streaming by clicking record button depicted in figure 5 and the recording is stored in Smartphone storage by record ID and verified by user. Another function is user can take part of streaming by clicking Take Picture depicted in figure 6 then the picture is stored and confirmed by user. When user wants to exit the application, user can click exit button.

User can watch the record on his/her Mobile Phone by pushing Load from Library button. After pushing the button, user enter to record list screen and choose the record as user preferences. User plays the record by clicking play button to play the record that has been chosen. To return the main menu, user can push back button.

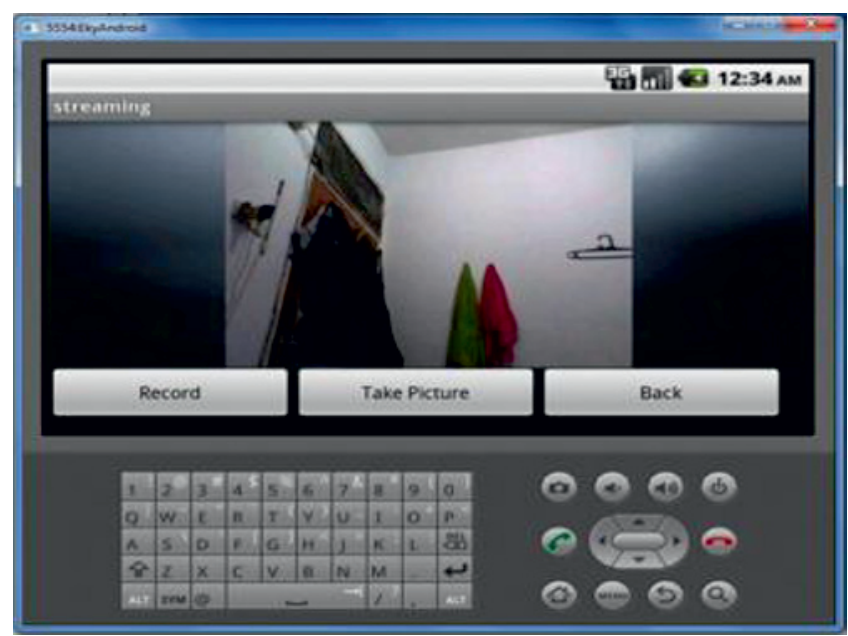

Fig. 5. Client Streaming Webcam Monitoring

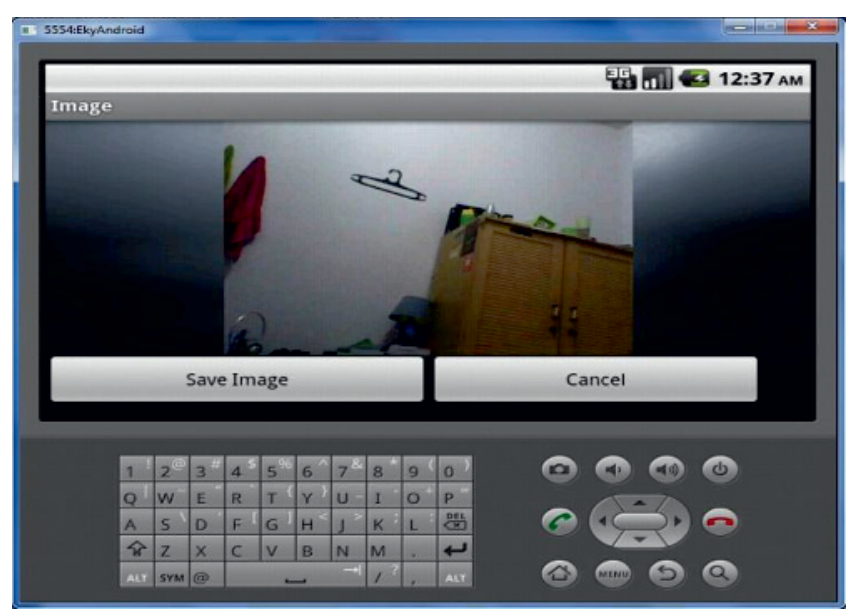

Fig. 4. Saving an image from webcam through client side

\subsection{Client Side}




\section{Conclusion}

Webcam monitoring application can help users to interact with the webcam on the computer by utilizing the Android-based Mobile Phone. It designed to assist and facilitate the user in monitoring the area with a webcam and access it directly to it. This simple application can be used by all Android-based mobile users. Webcam monitoring could make the user who previously not to monitor a certain area.

\section{References}

1. R. Logeswaran, Cost Effective Patient Location Monitoring System Using Webcams, J. Med. Syst. 33, 5, 399-407 (2009)

2. C.H Liu, C.C Fan, The Design of Remote Surveillance System for Digital Family, Fifth International Conference on Intelligent Information Hiding and Multimedia Signal Processing, 238-241 (2009)

3. J. Li, W. Hao, Research and Design of Embedded Network Video Monitoring System Based on Linux, International Conference on Computer Science and Software Engineering, 5 , 1310-1313 (2008)L. T. De Luca, Propulsion physics (EDP Sciences, Les Ulis, 2009)

4. L. Yang, L. Jiang, K. Yue, H. Pang, Design and Implementation of the Lab Remote Monitoring System Based on Embedded Web Technology, International Forum on Information Technology and Applications, 172-175 (2010)

5. W.K Wong, J.T.Y Liew, C.K Loo, W.K. Wong, Omnidirectional Surveillance System for Digital Home Security, International Conference on Signal Acquisition and Processing, 8-12 (2009) 